\title{
Osteoradionecrosis: A Review
}

\author{
Dr. Darshan K ${ }^{1}$, Dr. Chaithra Indumathi Vasudeva ${ }^{1}$, Dr. Chethana ${ }^{1}$, Dr. Preethi Poonja ${ }^{2 *}$, Dr. \\ Gowri Bhandarkar ${ }^{3}$, Dr. Prasanna Kumar Rao ${ }^{4}$, Dr. Raghavendra Kini ${ }^{5}$ \\ ${ }^{I}$ Intern, Department of Oral Medicine and Radiology, A.J Institute of Dental Sciences, Mangalore, Karnataka, \\ India \\ ${ }^{2}$ Assistant Professor, Department of Oral Medicine and Radiology, A.J Institute of Dental Sciences, Mangalore, \\ Karnataka, India \\ ${ }^{3}$ Reader, Department of Oral Medicine and Radiology, A.J Institute of Dental Sciences, Mangalore, Karnataka, \\ India \\ ${ }^{4}$ Professor, Department of Oral Medicine and Radiology, A.J Institute of Dental Sciences, Mangalore, \\ Karnataka, India \\ ${ }^{5}$ Professor and Head, Department of Oral Medicine and Radiology, A.J Institute of Dental Sciences, Mangalore, \\ Karnataka, India
}

*Corresponding Author: Dr. Preethi Poonja, Department of Oral Medicine and Radiology, A.J Institute of Dental Sciences, NH 66, Kuntikana, Mangalore, Karnataka, India. Email: preetipoonja@gmail.com

\begin{abstract}
Osteoradionecrosis(ORN) is a severe and delayed radiation-induced injury, characterized by bone tissue necrosis and failure to heal. Cases of osteoradionecrosis presents to the clinician with the features of pain, drainage and fistulation of the mucosa or skin related to exposed bone in the previously irradiated area. Better understanding of risk factors and pathophysiology may improve the ability of the clinician to prevent the occurrence and improve prognosis of the complication.
\end{abstract}

Keywords: Osteoradionecrosis, radiation, complications

\section{INTRODUCTION}

Osteoradionecrosis(ORN) of the jaw is one of the main complications of radiotherapy, which results in alteration of the shape and function of the oral cavity and jaw that causes substantial deterioration in patient quality of life. [1] There is necrosis of the bone due to obstruction of its blood supply. Patient who have received high dose of radiation therapy to the head and neck region, are predisposed to lifelong risk of osteoradionecrosis. [2] Although the incidence of ORN has declined recently with the advancement irradiation techniques and increased focus on predisposing factors. [1]

\section{DEFINITION}

In 1983, Marx defined ORN as 'an area more than $1 \mathrm{~cm}$ of exposed bone in a field of irradiation that failed to show any evidence of healing for at least 6 months'. [3]

According to the literature published in the last 15 years, ORN of the jaws is defined as exposed irradiated bone that fails to heal over a period of 3 months without evidence of persisting or recurrent tumor. At the time of diagnosis, the necrosis might involve the bone superficially or deeply. It might be a process that progresses slowly or an active progressive state that can lead to a pathological fracture. [3]

\section{ETIOLOGY}

- Doses above 50gy usually are required to cause this irreversible damage

- Usually, large radiation doses are required for osteoradionecrosis to occur (e.g. less than 3000-5000 cGy) [4]

- Dental extraction and dental trauma after radiation are predisposing factors

\section{PAthophysiology [5]}

Radiotherapy appears to cause ORN because it affects the small blood vessels of bone, inducing inflammation (endarteritis), which favors the generation of small thrombi that obliterate the vascular lumen and thus interrupt tissue perfusion. According to Marx, progressive hypoxia, hypovascularization and hypocellularity (3H Principle) are observed in the affected bone. [Figure1] 


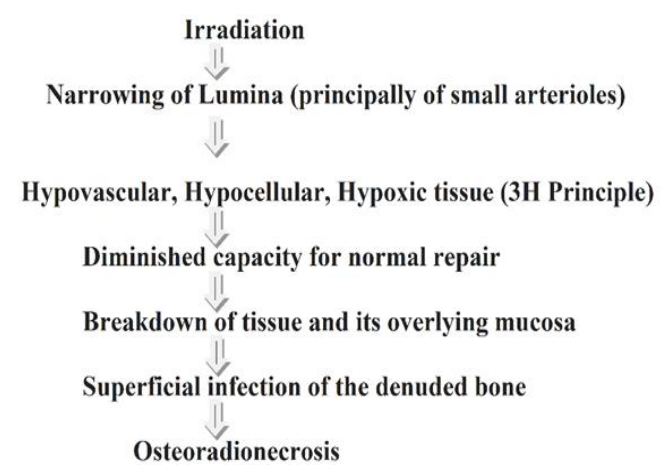

Figure1. Pathophysiology of osteoradionecrosis

Recently, a new theory known as the "fibroatrophic theory" has emerged, and proposes that fibroblast populations not only undergo total cellular depletion in response to radiation exposure, but also show a reduced ability to produce and secrete collagen into the surrounding tissue. This theory is based on the concept that osteoclasts suffer radiation damage earlier than the development of vascular alterations. Accordingly, the key event in the progression of ORN is the activation and dysregulation of fibroblastic activity that leads to atrophic tissue within a previously irradiated area. [1]

\section{Clinical Features [6]}

- Mandible most commonly affected because of micro anatomy and less vasculature

- Posterior mandible commonly affected than anterior

- Intense pain, with intermittent swelling and drainage

- Discoloration of the skin with extraoral fistula [Figure2]

- Loss of mucosa covering and exposure bone - Hallmark [Figure3]

- Exposed bone is grey to yellowish in color

- Asymptomatic dehiscence of mucosa, and loss of color

- Evidence of exposed bone

- Radiation caries can also be seen predominantly in the cervical region [Figure4]

- Tissue surrounding may be ulcerated from infection or recurrent tumor

- Trismus

- Fetid breath

- Elevated temperature

- Pathological fracture

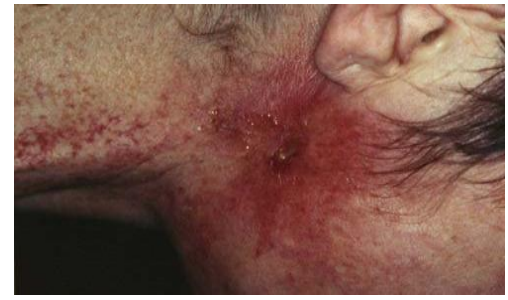

Figure2. Initial clinical signs of skin breakdown

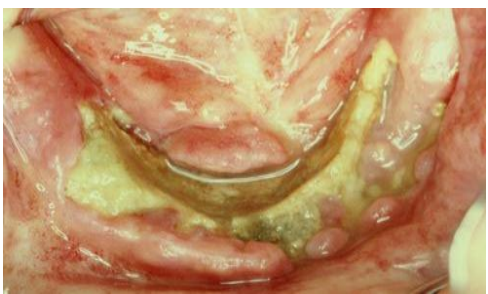

Figure3. Advanced mucosal breakdown with failure to heal with overlying necrotic bone

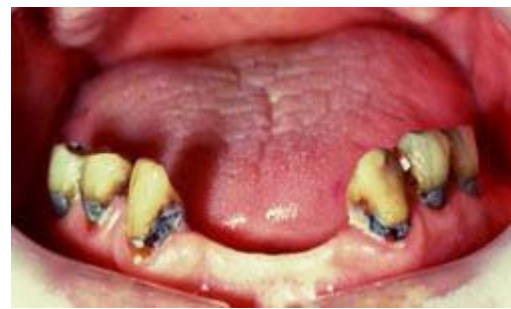

Figure4. Radiation Caries

\section{RADIOGRAPHIC FEATURES}

Conventional: Orthopantomogram (OPG) is the most frequently used imaging method for the diagnosis of ORN.

Early Changes of Bone: well defined area of bone resorption within the outer cortical plate

Later Changes: lytic or sclerotic or mixed. Periphery is ill defined and similar to that of osteomyelitis. If the lesion reaches the inferior border of the mandible, irregular resorption of this bony cortex often occurs leading to pathological fracture. [7] [Figure5]

CT, CBCT, MRI are the more advanced diagnostic modalities available.

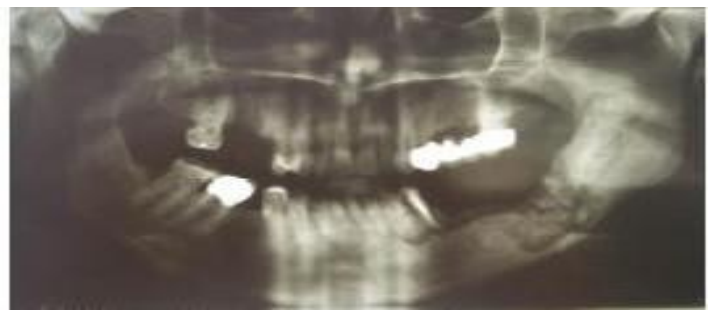

Figure5. Moth Eaten Appearance with Pathological Fracture

\section{MANAGEMENT}

Management of osteoradionecrosis includes conservative approach, radical method and surgical approach. 
Conservative approach to maintain the integrity of inferior border of mandible, keeping the site free of infection, and patient free of pain.

- "Conservative management" consists of local irrigation (saline solution, $\mathrm{NaHCO} 3$, or chlorhexidine $0.2 \%$ ), systemic antibiotics in acute infectious episodes, avoidance of irritants (tobacco, alcohol, denture use) and oral hygiene instruction. [5]

- Antibiotic medications should always be instituted after bacterial identification and sensitivity testing, and any delays in surgical treatment should be avoided. [6]

- Administration of parenteral antibiotics and fluids. Penicillin + metronidazole or clindamycin alone is recommended ${ }^{[8]}$ Use of narcotics and non-narcotic analgesics, hydration, nutrition [6]

Table1. Modified Marx Protocol in Treatment of ORN

\section{Prevention [9]}

exposed bone with nonhealing wound-30 HBO treatment and review. those patient, who responded well - further $10 \mathrm{HBO}$

\begin{tabular}{|l|l|}
\hline $\begin{array}{l}\text { Stage I: } \\
\text { nonrespondent }\end{array}$ & considered as stage II ORN \\
\hline Stage II & local debridement to induce fresh bleeding followed by 10 HBO \\
\hline Stage III & $\begin{array}{l}\text { Non-respondent stage II ORN or those [Patients with cutaneous fistula, pathologic } \\
\text { fracture or inferior border resorption. Total 30 HBO treatment followed by surgical } \\
\text { resection with stabilization of the remaining jaw by external fixation or IMF }\end{array}$ \\
\hline Stage III R & if reconstruction is required, then they enter stage III R - further 10 HBO \\
\hline
\end{tabular}

Prevention of osteoradionecrosis is considered before, during and after the therapy[Table2]

Table2. Prevention of ORN

\begin{tabular}{|l|l|}
\hline Before therapy & $\begin{array}{l}\text { - Extraction of non-restorable teeth, teeth with considerable periodontal disease, patients } \\
\text { with poor oral health and motivation } \\
\bullet \text { Radiation therapy after } 21 \text { days of extraction }\end{array}$ \\
\hline During therapy & $\begin{array}{l}\text { - No invasive treatment should be given } \\
\text { - No invasive treatments - pulpotomies, pulpectomies, endodontic treatments can be } \\
\text { followed }\end{array}$ \\
\hline After therapy & $\begin{array}{l}\text { - Avoid of denture use } \\
\text { - Reinforce oral hygiene, first } 4 \text { months are known as Golden Window Period, Necessary } \\
\text { oral surgical procedures can be done without HBO therapy } \\
\text { - Application of topical fluoride } \\
\text { - Salivary substitutes }\end{array}$ \\
\hline
\end{tabular}




\section{CONCLUSION}

Osteoradionecrosis can be a cruel blow to patients and their families who have been enduring radiotherapy for the treatment of cancer. Prevention of osteoradionecrosis by regular follow-up and early diagnosis should be the goal of every health care professional managing head and neck cancer patients. Improving radiotherapy protocols, multidisciplinary preventive care and reconstructive surgery can help to improve the quality of life of patients suffering from osteoradionecrosis.

\section{REFERENCES}

[1] New approach for treatment of osteoradionecrosis with Pentoxifylline and Tocopherol - Huan Fan, Soung Min Kim, Yun Ju Cho, Mi Young Eo, Suk Keun Lee, Kyung Mi Woo (29 September 2014)

[2] Burket's Text book of Oral Medicine - 12th Edition- PMPH-USA
[3] Osteoradionecrosis of the jaws: Definition, epidemiology, staging and clinical and radiological findings. A concise review Aristeidis Chronopoulos, Theodora Zarra, Michael Ehrenfeld, Sven Otto (25 June 2017)

[4] Osteoradionecrosis - Dr. Daniel J Bell, Dr.Yuranga Weerakkody et al

[5] Osteoradionecrosis of jaws: Clinico-therapeutic management: A literature review and update Koteswara Rao Nadella, Rama Mohan Kodali, Leela Krishna Guttikonda, Ashok Jonnalagadda (10 March 2015)

[6] Text book of Oral and Maxillofacial Surgery 3rd Edition by Neelima Malik -JAYPEE BROTHERS

[7] Principles and interpretation - Oral Radiology 6th Edition by White and Pharoah

[8] Oral and Maxillofacial Infections - 4th Edition by Topazian Goldberg Hupp

[9] American Journal of Oral medicine and radiology - Radiotherapy a Curse or Boon

Citation: Dr. Darshan K. Osteoradionecrosis: A Review. ARC Journal of Dental science. 2019; 4(2):14-17. doi:dx.doi.org/ 10.20431/2456-0030. 0402004.

Copyright: (c) 2019 Authors. This is an open-access article distributed under the terms of the Creative Commons Attribution License, which permits unrestricted use, distribution, and reproduction in any medium, provided the original author and source are credited. 